\title{
Research Paper: Effects of Online Attentional Bias Modification on Coronavirus Anxiety
}

\author{
Saeed Nasiry ${ }^{1^{*}}$ (D), Zahra Ameli² (D) \\ 1. Department of Clinical Psychology, School of Medicine, Shahid Beheshti University of Medical Sciences, Tehran, Iran. \\ 2. Department of Social Sciences, School of Letters and Humanities, Shahid Beheshti University, Tehran, Iran.
}

\begin{tabular}{|c|c|}
\hline $\begin{array}{l}\text { Use your device to scan } \\
\text { and read the article online }\end{array}$ & \\
\hline 口itrig & Chation: Nasiry, S., Ameli, A. (2021). Effects of Online Attentional Bias Modification on Coronavirus Anxiety. Journal of \\
\hline Expy & Practlce in Clinical Psychology, 9(2), 143-152. nttps://dol.org/10.32598/jpcp.9.2.139.2 \\
\hline 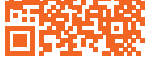 & doilhttps://doi.org/10.32598/jpcp.9.2.739.2 \\
\hline
\end{tabular}

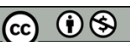

Article info:

Received: 14 Nov 2020

Accepted: 03 Feb 2021

Available Online: 01 Apr 2021

Keywords:

Attentional Bias Modification, Coronavirus anxiety, Covid-19, Dot-Probe task

\section{ABSTRACT}

Objective: The negative impacts of the Coronavirus Disease 2019 (COVID-19)outbreak on public mental health are enhancing the number of individuals requiring psychotherapy. Besides, anxiety is becoming more frequent than any other mental health issue among individuals. Similar to other anxiety types, coronavirus anxiety is associated with elevated attentional bias. The present study aimed to examine the effects of Attentional Bias Modification (ABM) on attentional bias towards coronavirus-related stimuli, and coronavirus anxiety.

Methods: This was a quasi-experimental study with pretest-posttest-follow-up and a control group design. The research participants were 37 adults (aged 18 to $38 \mathrm{y}$ ), who were randomly assigned to the experimental $(n=18)$ and $\operatorname{control}(n=19)$ groups. The experimental group received $\mathrm{ABM}$, while no intervention was delivered to the controls. Attentional bias and coronavirus disease anxiety were assessed at pretest, posttest, and 2 months follow-up stages using the DotProbe Task (DPT) and the Corona Disease Anxiety Scale (CDAS). The collected data were analyzed using two-way repeated-measures Analysis of Variance (ANOVA) and Fisher's least significant difference in SPSS v. 26.

Results: After receiving online ABM, the study participant's coronavirus-related attentional bias decreased, leading to a significant reduction in coronavirus anxiety $(\mathrm{P}<0.05)$. These significant changes were evident at the posttest and maintained until the follow-up step. However, no significant changes occurred in the control group $(\mathrm{P}>0.05)$.

Conclusion: The presented ABM could decrease coronavirus anxiety; thus, its online implementation is a suitable approach to treat individuals with this anxiety while observing social distancing.

\section{Corresponding Author:}

Saeed Nasiry, MSc.

Address: Department of Clinical Psychology, School of Medicine, Shahid Beheshti University of Medical Sciences, Tehran, Iran.

Tel: +98 (939) 8399387

E-mail:snasiry@sbmu.ac.ir 


\section{Highlights}

- Attentional Bias Modification (ABM) significantly reduced attentional bias towards corona-related stimuli in the study subjects.

- Reduced attentional bias led to decreased coronavirus anxiety.

- Online implementation of ABM was effective in improving coronavirus anxiety.

\section{Plain Language Summary}

People with anxiety tend to pay more attention to information related to the threat. For example, when encountering happy and angry faces, people with social anxiety pay more attention to angry faces. Similarly, people with coronavirus anxiety pay more attention to coronavirus-related information. This condition is called attentional bias and plays an important role in many psychological disorders. Attentional bias can be modified by various methods, known as Attentional Bias Modification (ABM). Researchers found that when an attentional bias associated with an anxiety type is reduced using ABM, the associated anxiety declines consequently. Therefore, this study aimed to examine whether ABM can reduce attentional bias to coronavirus-related information and decrease coronavirus anxiety. A web-based version of this intervention was delivered to 18 adults with moderate to severe coronavirus anxiety. Accordingly, their attentional bias and coronavirus anxiety were compared with 19 other adults who received no intervention. The study results suggested the efficacy of ABM in reducing coronavirus-related attentional bias and coronavirus anxiety. Coronavirus anxiety is increasing in the general population; thus, online ABM, as a brief intervention not requiring people to leave homes, can be a potential treatment for coronavirus anxiety.

\section{Introduction}

n March $13^{\text {th }}$ of 2020, the World Health Organization declared the outbreak of Coronavirus Disease 2019 (COVID-19) as a pandemic; such a condition affects personal, familial, and public biopsychological health (Jakovljevic, Bjedov, Jaksic \& Jakovljevic, 2020). This pandemic has influenced personal and communal behaviors and changed individuals' lifestyles by affecting priorities and altering the operation of the healthcare system (Fiorillo \& Gorwood, 2020). COVID-19 pandemic has caused a sudden increase in death rate, unemployment, stigmatization, loneliness, and separation from loved ones; these variables reduced individuals' psychological health (Marčinko, Jakovljević, Jakšić, Bjedov \& Mindoljević Drakulić, 2020). Even the mere exposure to information about COVID-19 leads to aggravated anxiety (Sorokowski et al., 2020). Currently, these data are strewn across all media, making it almost impossible to avoid them in daily life.

A study at the beginning of the COVID-19 outbreak indicated that more than half of China's general population has reported the mental impact of the outbreak to be moderate to severe. Additionally, about a third of the general population were suffering from moderate to severe anxiety (Wang et al., 2020). More recent studies have demonstrated that COVID-19-induced anxiety negatively predicts mental wellbeing (Ojiaku, Iorfa, Mefoh, Ezeuzo \& Odinko, 2020). Furthermore, the emotional impact of the pandemic can deteriorate the psychological health of patients with mental health disorders. This is because of their special vulnerability to stress; such conditions can exacerbate their existing mental health disorders (Yao, Chen \& Xu, 2020). These findings are not unexpected, also in the past, the outbreak of severe respiratory infections, like Severe Acute Respiratory Syndrome (SARS) had led to the increase of psychological distress in the general population (Leung et al., 2003).

The adverse impacts of the COVID-19 outbreak on public mental health are enhancing the frequency of individuals requiring psychotherapy (Marčinko et al., 2020). Prior research revealed that anxiety disorders are increasing more than any other mental issue (Huang \& Zhao, 2020). As a result, researchers intended to explore how coronavirus anxiety is affecting individuals and its management methods. Thus, previous empirical and theoretical knowledge regarding clinical anxiety is used to conduct novel studies and gain new understandings. For example, extensive empirical evidence supports the association between anxiety disorders and attentional bias. 
It was defined as a deficit in cognitive data processing, leading to increased selective attention towards threatrelated stimuli subsequently increasing an individual's anxiety (Bar-Haim, Lamy, Pergamin, BakermansKranenburg \& van Ijzendoorn, 2007; Van Bockstaele et al., 2014). Moreover, researchers are interested in exploring attentional bias towards information related to COVID-19 in individuals with relevant anxiety (Hagerty \& Williams, 2020; Cannito et al., 2020; Schudy et al., 2020).

A widely used instrument for measuring attentional bias concerning threat-related stimuli is the Dot-Probe Task (DPT) (Schmukle, 2005). In this task, stimuli pairs (lexical or pictorial) are side by side illustrated on a computer monitor. In each pair, one stimulus is threat-related and the other one is neutral. After a short while, the stimuli pair disappears and a probe (typically a dot; thus, the name DPT) is displayed in the place of one of the stimuli. Respondents are required to indicate the position of the probe by pushing a button. Reaction times to these probes are used to calculate the measures of attentional bias; faster reactions to probes replacing threat-related stimuli and slower reactions to probe replacing neutral stimuli, indicating higher attentional bias.

A study using the DPT demonstrated that like other anxiety types, coronavirus anxiety is associated with attentional bias. Therefore, the affected subjects could benefit from interventions targeting attentional bias (Cannito et al., 2020). Interventions designed to manipulate attentional bias are collectively recognized as Attentional Bias Modification (ABM). These interventions are usually developed by adjusting the methods of assessing attentional bias (Mathews \& MacLeod, 2002). For example, in the DPT, when the respondents are faced with pairs of threat-related and neutral stimuli, if the trials are designed in which the probe always appears in the place of the neutral stimuli, this encourages the participants to pay more attention towards the neutral stimuli; with the repetition of this training, their attentional bias towards threat-related stimuli decreases.

Some studies have examined the effects of psychotherapeutic interventions on general stress, anxiety, and depression during the COVID-19 pandemic (Chen, 2020; Li et al., 2020); however, to the best of our knowledge, no study has specifically investigated the effects of psychotherapeutic interventions on coronavirus e anxiety. There is growing empirical evidence supporting the efficacy of such methods as ABM in reducing attentional bias towards disorder-specific stimuli; consequently decreasing anxiety levels in different anxiety disorders
(Heeren, Mogoașe, Philippot \& McNally, 2015; MacLeod \& Clarke, 2015). Considering that attentional bias is present in coronavirus anxiety, ABM might be effective in treating this condition. This aim is achieved by reducing attentional bias towards corona-related stimuli. Furthermore, modifying cognitive biases can be successfully conducted remotely (Nasiry, Ameli \& Pezeshki, 2020). ABM is especially relevant in the current situation with the necessity of social distancing. However, the effectiveness of this intervention on coronavirus anxiety remains undiscovered.

In light of the necessity for further research on $\mathrm{CO}$ VID-19 and its associated mental health problems, the present study aimed to examine the effects of 8 sessions of online ABM on attentional bias towards corona-related stimuli. Besides, we explored the effects of ABM on coronavirus anxiety. According to the literature, we hypothesized that $\mathrm{ABM}$ can significantly reduce attentional bias and coronavirus anxiety.

\section{Materials and Methods}

The target population was all non-clinical Iranian adults. The study was conducted during quarantine; thus, all research procedures were conducted remotely. Furthermore, sampling was performed using online forums and social media platforms. The inclusion criteria of the study were as follows: having a minimum age of 18 years; generating mild to severe levels of coronavirus anxiety; being able to effectively use computers, and willingness to participate in the study. The exclusion criteria consisted of having severe physical illnesses, being diagnosed with mental health disorders, and discontinuing participation in the study before its completion. In total, 106 study participants completed the Corona Disease Anxiety Scale (CDAS). Of them, 49 subjects were scored $\geq 17$, indicating mild to severe coronavirus anxiety. Accordingly, 46 participants were randomly assigned to the experimental and control groups $(n=23 /$ group). Additionally, 5 and 4 participants from the experimental and control groups discontinued contribution to the study, respectively; thus, their data were excluded from the analysis. Eventually, the data obtained from 37 participants (21 females), aged 18-38 years (Mean $\pm \mathrm{SD}=$ $26.9 \pm 4.2$ ) were analyzed in the current study.

The following instruments were used in this study:

\section{Corona Disease Anxiety Scale (CDAS)}

Coronavirus anxiety was measured using CDAS. CDAS is a self-report questionnaire developed and vali- 
dated for measuring anxiety caused by the outbreak of COVID-19 in Iran (Alipour, Ghadami, Alipour \& Abdollahzadeh, 2020). It is comprised of 18 items and 2 factors, with the first 9 and second 9 items assessing psychological and physical symptoms, respectively. Each item is scored on a 4-point Likert-type scale, and the total score ranges from 0 to 54. Cronbach's alpha coefficient of 0.87 for the psychological symptoms, 0.86 for the physical symptoms, and 0.91 for the whole measure have established its internal consistency. Exploratory and confirmatory factor analyses have approved SDAS's structural validity; significant associations between its scores with the scores of the General Health Questionnaire-28 (GHQ-28) have supported the criterion validity of this measure.

\section{Dot-Probe Task (DPT)}

Attentional bias was measured using DPT, i.e., designed by MacLeod, Mathews \& Tata (1986). In this task, the research participants are presented with trials that initiate with a cross at the center of the screen on which they should fixate their gaze. After $500 \mathrm{~ms}$, the fixation cross disappears and a pair of stimuli are illustrated side by side. After another $500 \mathrm{~ms}$, both stimuli are removed and a probe (either $<$ or $>$ ) is randomly displayed in the place of one of the stimuli. Respondents should indicate the location of the probe as fast and accurately as possible, using the arrow keys on their keyboards. Stimuli pairs were comprised of one corona-related picture (e.g. masks, gloves, viruses, etc.) and one neutral picture (e.g. buildings, doors, windows, etc.), or two neutral pictures. Before conducting the study, pictures were rated from 0 to 100 by a sample of 78 individuals regarding their neutrality and relatedness to COVID-19. In the threatneutral pairs, the probe was presented 40 times in the location of corona-related stimuli (congruent trials) and 40 times in the location of the neutral stimuli (incongruent trials). In the neutral pairs, the probe was presented 40 times in either direction. Therefore, the task was comprised of 120 trials.

Reaction Times (RTs) to congruent, incongruent, and neutral trials were recorded and used to compute the measures of attentional bias. Concerning the standard version of DPT, attentional bias is calculated as the total RTs of incongruent trials minus the total RTs of congruent trials. However, previous research demonstrated that the standard version of this instrument has poor psychometric properties; therefore, this defect might lead to inconsistent results across different studies (Cristea, Kok \& Cuijpers, 2015; Schmukle, 2005). In response to this issue, a new version of this instru- ment with a response-based approach to computing attentional bias measures was developed, with high levels of reliability and validity (Evans \& Britton, 2018). In the response-based version of the DPT, i.e., used in this study, the participant's response to each trial was separately referenced against a mean reference RT. To attain the measures of attentional bias, the individual RTs of congruent trials were subtracted from the mean RTs of incongruent trials, as a reference. Responses with RTs lower than the reference RT constitute the distribution of attentional vigilance responses; those with RTs higher than the reference RT form the distribution of attentional avoidance responses. Then, the average RT of vigilance responses can be used as a measure of vigilant attentional bias (towards coronavirus-related stimuli). Consequently, the mean RTs of avoidance responses can be considered as a measure of avoidant attentional bias (away from coronavirus-related stimuli).

\section{Attentional Bias Modification (ABM)}

This intervention was adapted from the training that $\mathrm{Zi}$ aee, Fadardi, Cox \& Yazdi (2016). ABM was designed for modifying attentional bias towards drug-related stimuli by adjusting a modified Stroop task. Before the onset of the study, a series of 56 words and 56 pictures were selected as potential stimuli (half of which were coronavirus-related, e.g. masks, gloves, \& viruses; the other half were neutral, e.g. buildings, doors, \& windows). These potential stimuli were rated from 0 to 100 by a sample of 78 individuals regarding their neutrality and relatedness to COVID-19. Moreover, for each set of neutral and coronavirus-related stimuli, 14 words and 14 pictures with the highest rates were chosen. In this version of the training, coronavirus-related and neutral stimuli were used in lexical and pictorial formats. Subsequently, the study participants were requested to itemize the color of either the background or the border of each stimulus with the highest speed and the least number of errors, using their computer keyboards. In the first 4 sessions, single stimuli were randomly presented to the study participants. Additionally, they had to ignore the contents of the stimuli and specify the color of their backgrounds or borders. In the second 4 sessions, paired stimuli (one coronavirus-related \& one neutral, side by side with random placements) were presented; the research participants had to direct their attention towards the neutral stimuli and identify the color of their backgrounds or borders. Each session was designed to be harder than the previous one. Furthermore, the criteria for moving from one session to the next were response time to each stimulus and the total errors. The details of ABM sessions are presented in Table 1. 
Table 1. The contents of Attentional Bias Modification (ABM) sessions

\begin{tabular}{|c|c|c|c|c|c|}
\hline \multirow{2}{*}{ Sessions } & \multicolumn{3}{|c|}{ Contents } & \multicolumn{2}{|c|}{ Success Criteria } \\
\hline & Stimulus & Presentation & Response & RT & NE \\
\hline 1 & Pictorial & Single & Background color & $<1$ second & $<4$ errors \\
\hline 2 & Lexical & Single & Background color & $<1$ second & $<4$ errors \\
\hline 3 & Pictorial & Single & Border color & $<1$ second & $<3$ errors \\
\hline 4 & Lexical & Single & Border color & $<1$ second & $<3$ errors \\
\hline 5 & Pictorial & Paired & Background color & $<1$ second & $<3$ errors \\
\hline 6 & Lexical & Paired & Background color & $<1$ second & $<3$ errors \\
\hline 7 & Pictorial & Paired & Border color & $<1$ second & $<2$ errors \\
\hline 8 & Lexical & Paired & Border color & $<1$ second & $<2$ errors \\
\hline
\end{tabular}

RT: Response Time; NE: Number of Errors

PRACTICE II PLYNICAL PSYH $\cong$ LOGY

This was a quasi-experimental study with a pretestposttest-follow-up and a control group design. The study participants were instructed to sit in front of a computer in a quiet and private room, with an ideal $60 \mathrm{~cm}$ distance from the monitor. They completed the DPT at pretest (CDAS was completed at the sampling stage). Accordingly, the intervention was initiated in the experimental group. In total, eight 20-minute daily ABM sessions were conducted. Posttest measurements using DPT and CDAS were conducted right after the last session of ABM in the experimental group, and 8 days after the pretest step in the control group. Follow-up measurements were conducted after 2 months. The obtained data were analyzed using two-way repeated-measures Analysis of Variance (ANOVA) and Fisher's Least Significant Difference (LSD) in SPSS v. 26.

This study was conducted per all ethical guidelines. The study protocol was per the ethical standards of the declaration of Helsinki in 1975 (i.e., revised in 2000). The objectives, protocol, and implications of the study were explained to all study participants, and informed consent forms were obtained from them. Additionally, the study participants were ensured about the confidentiality of their information. The study subjects were also allowed to discontinue cooperation with the research at any stage. Eventually, the obtained results and their interpretations were explained to the explored participants in a simplified manner, and they were appreciated for their contributions to this research.

\section{Results}

To analyze the research participants' demographic and clinical baseline data, Independent Samples t-test (for the quantitative data), and Chi-squared test (for the qualitative data_) were employed. The relevant data, revealed no significant difference between the study groups, in age, gender, educational level, and the severity of coronavirus anxiety $(\mathrm{P}>0.05)$ (Table 2$)$.

To test the study's hypothesis regarding the effects of $\mathrm{ABM}$ on coronavirus-related attentional bias and coronavirus anxiety, two-way repeated-measures ANOVA was conducted to test the significance of the differences in scores between the experimental and control groups at multiple measurements. In this analysis, the within-subjects factor was time, the between-subjects factor was group, and the dependent variables were the research participants' scores on CDAS and DPT (Table 3). The relevant results indicated that the effects of time, group, and time*group interaction were significant on the CDAS and DPT scores $(\mathrm{P}<0.05)$. In other words, there existed significant differences between the study groups at multiple measurements respecting coronavirus-related attentional bias and coronavirus anxiety (Table 4).

After significant effects were revealed in the analysis, posthoc pairwise comparisons were conducted using Fisher's LSD to identify the exact points of significant changes in the data. The relevant results demonstrated that significant alternations only occurred in the experimental group. Besides, this change was evident in their coronavirus-related attentional bias and coronavi- 
Table 2. Demographic and clinical characteristics of the study participants at baseline

\begin{tabular}{|c|c|c|c|c|}
\hline \multirow{2}{*}{ Variables } & \multicolumn{2}{|c|}{ Groups } & \multirow{2}{*}{ Statistics } & \multirow{2}{*}{$\mathbf{P}$} \\
\hline & Experimental & Control & & \\
\hline Age (y), Mean $\pm S D$ & $25.86 \pm 4.61$ & $28.02 \pm 3.87$ & $\mathrm{t}=1.54$ & 0.13 \\
\hline Gender (male/female), N & $9 / 9$ & $7 / 12$ & $x^{2}=0.65$ & 0.41 \\
\hline Education (diploma/undergraduate/graduate), $\mathrm{N}$ & $3 / 9 / 6$ & $2 / 12 / 5$ & $x^{2}=0.69$ & 0.70 \\
\hline Coronavirus anxiety (moderate/severe), $\mathrm{N}$ & $13 / 5$ & $12 / 7$ & $x^{2}=0.34$ & 0.55 \\
\hline
\end{tabular}

rus anxiety $(\mathrm{P}<0.05)$. As Table 5 presents, from the two measures of attentional bias, only vigilant attentional bias significantly changed at posttest, i.e., maintained until follow-up. Furthermore, no significant association was observed in neither group's avoidant attentional bias and coronavirus anxiety $(\mathrm{P}>0.05)$. Moreover, the scores of CDAS and its subscales significantly changed in the experimental group. Concerning the total scale and the psychological symptoms subscale, these changes were observed at posttest and maintained until follow-up; however, for the physical symptoms subscale, a significant change only occurred at follow-up. No significant change was observed in the control group in this area $(\mathrm{P}>0.05)$.

\section{Discussion}

This study was the first attempt for examining the effects of ABM on attentional bias towards coronavirusrelated stimuli and coronavirus anxiety. For this purpose, 8 online ABM sessions were delivered to a non-clinical sample of adults with moderate to severe coronavirus anxiety. Accordingly, the effects of the intervention on this group were compared with a control group that received no intervention.

The present research findings demonstrated that ABM can successfully modify attentional bias towards coronavirus-related stimuli. The study participants who received ABM indicated significantly reduced levels of vigilant attentional bias in the DPT at posttest and follow-up phases, compared with the pretest step. Similar changes were not observed in the control group. This result was consistent with those of previous studies supporting the efficacy of ABM in the manipulation of selective processing of information. Based on the cognitive theories of anxiety, individuals with high levels of anxiety present a continuous attentional bias towards threat-related stimuli; however, individuals with low levels of anxiety avoid threat (Mogg \& Bradley, 2018). Attentional bias towards threat is a stable trait playing a causal role in anxiety; therefore, interventions that target

Table 3. Mean $\pm S D$ scores of the measures for the studied dependent variables

\begin{tabular}{|c|c|c|c|c|c|c|}
\hline \multirow{2}{*}{ Groups } & \multirow{2}{*}{ Time } & \multicolumn{3}{|c|}{ CDAS } & \multicolumn{2}{|c|}{ Dot Probe Task } \\
\hline & & Total & PSY & PHY & VAB & AAB \\
\hline \multirow{3}{*}{ Experimental } & Pretest & $19.21 \pm 12.44$ & $12.16 \pm 7.29$ & $7.05 \pm 5.38$ & $163.40 \pm 39.27$ & $11.95 \pm 5.16$ \\
\hline & Posttest & $11.86 \pm 8.17$ & $7.33 \pm 4.52$ & $4.53 \pm 3.94$ & $21.12 \pm 9.73$ & $10.61 \pm 5.80$ \\
\hline & Follow-up & $8.45 \pm 5.58$ & $5.09 \pm 2.93$ & $3.36 \pm 2.81$ & $17.88 \pm 7.06$ & $14.11 \pm 7.15$ \\
\hline \multirow{3}{*}{ Control } & Pretest & $20.85 \pm 10.41$ & $13.26 \pm 6.96$ & $7.99 \pm 4.87$ & $159.58 \pm 36.07$ & $10.90 \pm 6.58$ \\
\hline & Posttest & $19.67 \pm 11.09$ & $14.55 \pm 7.53$ & $8.34 \pm 5.66$ & $171.77 \pm 41.75$ & $12.48 \pm 6.27$ \\
\hline & Follow-up & $21.13 \pm 11.34$ & $13.42 \pm 6.25$ & $8.71 \pm 5.43$ & $168.20 \pm 39.80$ & $12.33 \pm 5.89$ \\
\hline
\end{tabular}

CDAS: Corona Disease Anxiety Scale; PSY: Psychological Symptoms; PHY: Physical Symptoms; VAB: Vigilant Attentional Bias; AAB: Avoidant Attentional Bias 
Table 4. The two-way repeated-measures ANOVA data

\begin{tabular}{|c|c|c|c|c|c|c|c|}
\hline Variables & Sources & sS & df & MS & $\mathbf{F}$ & $\mathbf{P}$ & $\eta_{p}^{2}$ \\
\hline \multirow{5}{*}{ VAB } & Time & 194.05 & 2 & 97.03 & 14.86 & $<0.001$ & 0.30 \\
\hline & Time*Group & 223.49 & 2 & 111.75 & 17.11 & $<0.001$ & 0.33 \\
\hline & Error & 457.14 & 70 & 6.53 & & & \\
\hline & Group & 591.36 & 1 & 591.36 & 13.03 & $<0.001$ & 0.27 \\
\hline & Error & 1588.49 & 35 & 45.39 & & & \\
\hline \multirow{5}{*}{$A A B$} & Time & 16.48 & 2 & 8.24 & 0.65 & 0.53 & 0.02 \\
\hline & Time*Group & 42.51 & 2 & 21.26 & 1.67 & 0.20 & 0.05 \\
\hline & Error & 889.60 & 70 & 12.70 & & & \\
\hline & Group & 3.76 & 1 & 3.76 & 0.30 & 0.59 & 0.00 \\
\hline & Error & 439.96 & 35 & 12.57 & & & \\
\hline \multirow{5}{*}{ TCDA } & Time & 229.19 & 2 & 114.59 & 10.74 & $<0.001$ & 0.24 \\
\hline & Time*Group & 606.85 & 2 & 303.43 & 28.49 & $<0.001$ & 0.49 \\
\hline & Error & 745.53 & 70 & 10.65 & & & \\
\hline & Group & 439.45 & 1 & 439.45 & 9.78 & $<0.01$ & 0.22 \\
\hline & Error & 1572.26 & 35 & 44.92 & & & \\
\hline \multirow{5}{*}{ PSY } & Time & 86.11 & 2 & 43.06 & 12.09 & $<0.001$ & 0.26 \\
\hline & Time*Group & 213.53 & 2 & 91.77 & 25.78 & $<0.001$ & 0.46 \\
\hline & Error & 249.27 & 70 & 3.56 & & & \\
\hline & Group & 197.42 & 1 & 197.42 & 11.89 & $<0.01$ & 0.25 \\
\hline & Error & 581.35 & 35 & 16.61 & & & \\
\hline \multirow{5}{*}{ PHY } & Time & 48.80 & 2 & 24.40 & 9.17 & $<0.001$ & 0.21 \\
\hline & Time*Group & 78.37 & 2 & 39.19 & 14.73 & $<0.001$ & 0.30 \\
\hline & Error & 186.29 & 70 & 2.66 & & & \\
\hline & Group & 93.76 & 1 & 93.76 & 7.66 & $<0.01$ & 0.18 \\
\hline & Error & 428.47 & 35 & 12.24 & & & \\
\hline
\end{tabular}

VAB:Vigilant Attentional Bias; AAB: Avoidant Attentional Bias; TCDA: Total Corona Disease Anxiety; PSY: Psychological Symptoms; PHY: Physical Symptoms; SS: Sum of Squares; df: Degrees of Freedom; MS: Mean Square; $\eta_{\mathrm{p}}^{2}$ : Partial Eta Squared

attentional bias towards threat and reduce it should also decline anxiety (MacLeod \& Clarke, 2015).

Contrary findings were also observed in the literature on ABM; however, this data inconsistency could be attributed to the point that those findings are often ob- served in the studies that implement a single session intervention; apply psychometrically unreliable instruments for measuring attentional bias; have large rates of dropout; or have typical methodological problems, like using the same cognitive task for both assessment and modification of attentional bias (Everaert, Mogoaşe, 
Table 5. The mean differences of studied variables in Fisher's Least Significant Difference (LSD)

\begin{tabular}{ccccccc}
\hline \multirow{2}{*}{ Group } & \multirow{2}{*}{ Comparison } & \multicolumn{2}{c}{ CDAS } & & \multicolumn{2}{c}{ Dot Probe Task } \\
\cline { 3 - 7 } & & Total & PSY & PHY & VAB & AAB \\
\hline \multirow{3}{*}{ Experimental } & Pretest \& Posttest & $-7.35^{*}$ & $-4.83^{*}$ & -2.52 & $-142.28^{*}$ & -1.34 \\
& Pretest \& Follow-up & $-10.76^{*}$ & $-7.07^{*}$ & $-3.69^{*}$ & $-145.52^{*}$ & 2.16 \\
& Posttest \& Follow-up & -3.41 & -2.24 & -1.17 & -3.24 & 3.50 \\
& Pretest \& Post-test & -1.18 & 1.29 & 0.35 & 12.19 & 1.58 \\
Control & Pretest \& Follow-up & 0.28 & 0.16 & 0.72 & 8.62 & 1.43 \\
& Posttest \& Follow-up & 1.46 & -1.13 & 0.37 & -3.57 & -0.15 \\
\hline
\end{tabular}

PRACTICE in PSINCAL PSY $\oplus$ LOGY

CDAS: Corona Disease Anxiety Scale; PSY: Psychological Symptoms; PHY: Physical Symptoms; VAB: Vigilant Attentional Bias; AAB: Avoidant Attentional Bias.

"The mean difference is significant at the 0.05 level.

David \& Koster, 2015; Mogg, Waters \& Bradley, 2017; Wen et al., 2020). The present study employed a multisession implementation of ABM, a psychometrically sound version of the DPT, and different tasks for the assessment (i.e., response-based DPT) and the manipulation (i.e., modified Stroop task) of attentional bias. Unlike vigilant attentional bias, avoidant attentional bias was not significantly influenced by ABM; however, this measure of attentional bias was not associated with the severity of coronavirus anxiety. Thus, its unaffectedness by ABM might not compromise the efficacy of ABM on coronavirus anxiety.

Consistent with this assumption, this research signified that ABM successfully reduced coronavirus anxiety. This was observed as a significant reduction in the experimental group's scores of CDAS. Besides, no significant change was observed in the controls. These results supported the study hypothesis. Furthermore, these data were consistent with the literature demonstrating that attentional bias plays a causal role in the etiology, maintenance, and severity of anxiety. Thus, the effects of ABM on attentional bias and anxiety were interrelated. In other words, ABM successfully reduced attentional bias; accordingly, it effectively decreased anxiety (Mogg et al., 2017). Further examination of the experimental group's CDAS scores revealed that their total scores and psychological symptoms scores significantly decreased at posttest and follow-up stages, compared to the pretest step; however, their physical symptoms scores significantly decreased only at follow-up, compared with pretest and posttest stages. This finding suggests that the effects of
$\mathrm{ABM}$ on the physical symptoms of coronavirus anxiety occur gradually and can remain stable over time.

There were certain limitations to this study. Due to the COVID-19 quarantine, the study measurements and intervention were conducted online and indirectly. Thus, the only criterion for the severity of coronavirus anxiety in the participants was their scores on the self-report questionnaire; however, examinations by clinical experts might provide a more precise evaluation of this condition, and are recommended to be used when possible. Furthermore, in the current study, only implementing a 2-month follow-up was possible; accordingly, it only confirms the stability of the intervention effects over a short time. Therefore, implementing longer follow-ups in future studies for assessing the maintenance of the intervention effects over the long term is necessary.

\section{Conclusion}

Despite the aforementioned limitations, the present study provided rudimentary support for the efficacy of an online implementation of ABM in reducing attentional bias towards coronavirus-related stimuli and decreasing coronavirus anxiety. Considering the growing need for mental health assistance due to the COVID-19 pandemic, and with the necessity of social distancing, $\mathrm{ABM}$, as an intervention that can be delivered online and successfully ameliorates coronavirus anxiety, is worth considering by the mental health and clinical experts. Nevertheless, this was only the first attempt in examining the effects of $\mathrm{ABM}$ on coronavirus anxiety; thus, 
further research is necessary for the replication of the obtained results.

\section{Ethical Considerations}

\section{Compliance with ethical guidelines}

The participants were informed of the purpose of the research and its implementation stages. A written consent has been obtained from the subjects. They were also assured about the confidentiality of their information and were free to leave the study whenever they wished, and if desired, the research results would be available to them. The Helsinki Convention was also observed.

\section{Funding}

This research did not receive any grant from funding agencies in the public, commercial, or non-profit sectors.

\section{Authors' contributions}

Conceptualization, design, methodology, and sampling: Saeed Nasiry; Data curation and analysis: Zahra Ameli; Drafting, editing, reviewing, and revising the article: Both authors.

\section{Conflict of interest}

The authors declared no conflicts of interest.

\section{References}

Alipour, A., Ghadami, A., Alipour, Z., \& Abdollahzadeh, H. (2020). [Preliminary validation of the Corona Disease Anxiety Scale (CDAS) in the Iranian sample (Persian)]. Quarterly Journal of Health Psychology, 8(23), 163-75. http:// hpj.journals. pnu.ac.ir/article_6571_en.html

Bar-Haim, Y., Lamy, D., Pergamin, L., Bakermans-Kranenburg, M. J., \& van Ijzendoorn, M. H. (2007). Threat-related attentional bias in anxious and nonanxious individuals: A meta-analytic study. Psychological Bulletin, 133(1), 1-24 [DOI:10.1037/0033-2909.133.1.1] [PMID]

Cannito, L., Di Crosta, A., Palumbo, R., Ceccato, I., Anzani, S., \& La Malva, P., et al. (2020). Health anxiety and attentional bias toward virus-related stimuli during the COVID-19 pandemic. Scientific Reports, 10(1), 16476. [DOI:10.1038/s41598020-73599-8] [PMID] [PMCID]

Chen S. (2020). An online solution focused brief therapy for adolescent anxiety during the novel coronavirus disease (COVID-19) pandemic: A structured summary of a study protocol for a randomised controlled trial. Trials, 21(1), 402. [DOI:10.1186/s13063-020-04355-6] [PMID] [PMCID]
Cristea, I. A., Kok, R. N., \& Cuijpers, P. (2015). Efficacy of cognitive bias modification interventions in anxiety and depression: Meta-analysis. The British Journal of Psychiatry, 206(1), 7-16. [DOI:10.1192/bjp.bp.114.146761] [PMID]

Evans, T. C., \& Britton, J. C. (2018). Improving the psychometric properties of dot-probe attention measures using responsebased computation. Journal of Behavior Therapy and Experimental Psychiatry, 60, 95-103. [DOI:10.1016/j.jbtep.2018.01.009] [PMID]

Everaert, J., Mogoaşe, C., David, D., \& Koster, E. H. W. (2015) Attention bias modification via single-session dot-probe training: Failures to replicate. Journal of Behavior Therapy and Experimental Psychiatry, 49(Pt A), 5-12. [DOI:10.1016/j. jbtep.2014.10.011] [PMID]

Fiorillo, A., \& Gorwood, Ph. (2020). The consequences of the COVID-19 pandemic on mental health and implications for clinical practice. European Psychiatry, 63(1), e32. [DOI:10.1192/j.eurpsy.2020.35] [PMID] [PMCID]

Hagerty, S. L., \& Williams, L. M. (2020). The impact of COVID-19 on mental health: The interactive roles of brain biotypes and human connection. Brain, Behavior, E Immunity-Health, 5 100078. [DOI:10.1016/j.bbih.2020.100078] [PMID] [PMCID]

Heeren, A., Mogoașe, C., Philippot, P., \& McNally, R. J. (2015). Attention bias modification for social anxiety: A systematic review and meta-analysis. Clinical Psychology Review, 40, 7690. [DOI:10.1016/j.cpr.2015.06.001] [PMID]

Huang, Y., \& Zhao, N. (2020). Generalized anxiety disorder, depressive symptoms and sleep quality during COVID-19 outbreak in China: A web-based cross-sectional survey. Psychiatry Research, 288, 112954. [DOI:10.1016/j.psychres.2020.112954] [PMID] [PMCID]

Jakovljevic, M., Bjedov, S., Jaksic, N., \& Jakovljevic, I. (2020). COVID-19 pandemia and public and global mental health from the perspective of global health security. Psychiatria Danubina, 32(1), 6-14. [DOI:10.24869/psyd.2020.6] [PMID]

Leung, G. M., Lam, T. H., Ho, L. M., Ho, S. Y., Chan, B. H. Y., \& Wong, I. O. L., et al. (2003). The impact of community psychological responses on outbreak control for severe acute respiratory syndrome in Hong Kong. Journal of Epidemiology \& Community Health, 57(11), 857-63. [DOI:10.1136/jech.57.11.857] [PMID] [PMCID]

MacLeod, C., \& Clarke, P. J. F. (2015). The attentional bias modification approach to anxiety intervention. Clinical Psychological Science, 3(1), 58-78. [DOI:10.1177/2167702614560749]

MacLeod, C., Mathews, A., \& Tata, P. (1986). Attentional bias in emotional disorders. Journal of Abnormal Psychology, 95(1), 15-20. [DOI:10.1037/0021-843X.95.1.15] [PMID]

Marčinko, D., Jakovljević, M., Jakšić, N., Bjedov, S., \& Mindoljević Drakulić, A. (2020). The importance of psychodynamic approach during COVID-19 pandemic. Psychiatria Danubina, 32(1), 15-21. [DOI:10.24869/psyd.2020.15] [PMID]

Mathews, A., \& MacLeod, C. (2002). Induced processing biases have causal effects on anxiety. Cognition and Emotion, 16(3), 331-54. [DOI:10.1080/02699930143000518]

Mogg, K., \& Bradley, B. P. (2018). Anxiety and threat-related attention: Cognitive-motivational framework and treatment Trends in Cognitive Sciences, 22(3), 225-40. [DOI:10.1016/j. tics.2018.01.001] [PMID] 
Mogg, K., Waters, A. M., \& Bradley, B. P. (2017). Attention Bias Modification (ABM): Review of effects of multisession ABM training on anxiety and threat-related attention in high-anxious individuals. Clinical Psychological Science, 5(4), 698-717. [DOI:10.1177/2167702617696359] [PMID] [PMCID]

Nasiry, S., Ameli, Z., \& Pezeshki, P. (2020). Online cognitive bias modification of interpretation for children with obsessivecompulsive disorder. Practice in Clinical Psychology, 8(4), 32534. [DOI:10.32598/jpcp.8.4.739.1]

Ojiaku, Ch. M., Iorfa, S., Mefoh, Ph. C., Ezeuzo, O., \& Odinko, I. Ch. (2020). COVID-19-induced anxiety and Covid-19 precautionary measures as predictors of mental wellbeing of Nigerians. International Journal of Behavioral Sciences, 14(3), 149-54. [DOI:10.30491/IJBS.2020.239210.1342]

Schmukle, S. C. (2005). Unreliability of the dot probe task. European Journal of Personality, 19(7), 595-605. [DOI:10.1002/per.554]

Schudy, A., Żurek, K., Wiśniewska, M., Piejka, A., Gawẹda, Ł., \& Okruszek, Ł. (2020). Mental well-being during pandemic: The role of cognitive biases and emotion regulation strategies in risk perception and affective response to COVID-19. Frontiers in Psychiatry, 11, 589973. [DOI:10.3389/fpsyt.2020.589973] [PMID] [PMCID]

Sorokowski, P., Groyecka, A., Kowal, M., Sorokowska, A., Białek, M., \& Lebuda, I., et al. (2020). Can information about pandemics increase negative attitudes toward foreign groups? A case of COVID-19 outbreak. Sustainability, 12(12), 4912. [DOI:10.3390/su12124912]

Van Bockstaele, B., Verschuere, B., Tibboel, H., De Houwer, J., Crombez, G., \& Koster, E. H. W. (2014). A review of current evidence for the causal impact of attentional bias on fear and anxiety. Psychological Bulletin, 140(3), 682-721. [DOI:10.1037/ a0034834] [PMID]

Wang, C., Pan, R., Wan, X., Tan, Y., Xu, L., \& Ho, C. S., et al. (2020). Immediate psychological responses and associated factors during the initial stage of the 2019 coronavirus disease (COVID-19) epidemic among the general population in China. International Journal of Environmental Research and Public Health, 17(5), 1729. [DOI:10.3390/ijerph17051729] [PMID] [PMCID]

Wen, S., Larsen, H., Boffo, M., Grasman, R. P. P. P., Pronk, T., \& van Wijngaarden, J. B. G., et al. (2020). Combining web-based attentional bias modification and approach bias modification as a self-help smoking intervention for adult smokers seeking online help: Double-blind randomized controlled trial. JMIR Mental Health, 7(5), e16342. [DOI:10.2196/16342] [PMID] [PMCID]

Yao, H., Chen, J. H., \& Xu, Y. F. (2020). Patients with mental health disorders in the COVID-19 epidemic. The Lancet Psychiatry, 7(4), E21. [DOI:10.1016/S2215-0366(20)30090-0]

Ziaee, S. S., Fadardi, J. S., Cox, W. M., \& Yazdi, S. A. A. (2016). Effects of attention control training on drug abusers' attentional bias and treatment outcome. Journal of Consulting and Clinical Psychology, 84(10), 861-73. [DOI:10.1037/a0040290] [PMID] 